\title{
Does Chronic Treatment with Oral Anticoagulants Ameliorate the Clinical Course of Severe Acute Respiratory Syndrome Coronavirus 2 (SARS-CoV-2) Infection in Coronavirus Disease 2019 (COVID-19)?
}

\author{
Job Harenberg, MD ${ }^{1,2}$ Rupert Bauersachs, $\mathrm{MD}^{3}$ \\ ${ }^{1}$ Ruprecht Karls University, Heidelberg, Germany \\ 2 Doasense $\mathrm{GmbH}$, Heidelberg, Germany \\ ${ }^{3}$ Department of Vascular Medicine, Klinikum Darmstadt GmbH, \\ Darmstadt, Germany \\ ${ }^{4}$ Department of Medicine and Surgery, University of Insubria, Varese, Italy
}

Semin Thromb Hemost 2021;47:338-340.

The risk of ischemic stroke in patients with nonvalvular atrial fibrillation (NVAF) increases from $1.5 \%$ at 50 to 59 years of age to $23.5 \%$ at 80 to 89 years of age, ${ }^{1}$ and this is reflected in the $\mathrm{CHA}_{2} \mathrm{DS}_{2}$-VASc score. ${ }^{2}$ The incidence of venous thromboembolism (VTE) also increases from $0.1 \%$ by $1 \%$ per year at 50 years of age. ${ }^{3}$ However, the incidence of NVAF was not higher in older patients hospitalized with coronavirus disease 2019 (COVID-19). Mortality rates in patients with COVID-19were associated with older age, ${ }^{4}$ as well as diabetes and hypertension, which are also risk factors for adverse outcomes in NVAF. ${ }^{5}$ Surprisingly, mortality of COVID-19 patients was not associated with a history of NVAF and VTE. The American Heart Association has established a registry "Get With The Guidelines (GWTG)" to collect data on COVID-19 patients hospitalized with NVAF, coronary artery disease, heart failure, stroke, and in-hospital cardiac arrest. Active viral or bacterial infections are being documented during hospitalization, including emerging infectious diseases such as COVID-19. ${ }^{6}$

Patients with NVAF and VTE are preferentially treated with a fixed dose of direct oral anticoagulants (DOACs) or with vitamin $\mathrm{K}$ antagonists (VKA) adjusted according to the international normalized ratio (INR) to prevent ischemic stroke and recurrent VTE. ${ }^{7}$

Therefore, given the preceding background, we propose that patients on long-term oral anticoagulant treatment may be protected from a more severe course of COVID-19 disease (-Fig. 1). Differences between VKA and DOACs and between factor Xa inhibitors (apixaban, edoxaban, and rivaroxaban) and the thrombin inhibitor dabigatran may affect the relative severity of COVID-19, although this needs

published online September 3, 2020
Issue Theme Maintaining Hemostasis and Preventing Thrombosis in COVID-19 -Part II; Guest Editors: Emmanuel J. Favaloro, PhD, FFSc (RCPA) and Giuseppe Lippi, MD.

to be shown. The lack of age-dependent mortality in COVID19 patients with NVAF or a history of VTE may be explained by treatment with VKA or DOAC before hospitalization and may open new perspectives on disease prevention and treatment.

Effects of anticoagulants need to be determined rapidly and accurately to ascertain whether they should be administered further and to avoid hemorrhage in COVID-19 patients with NVAF and VTE. Rapid testing for anticoagulant status may also be useful to determine whether a switch from VKA or DOACs to heparin or low molecular weight heparin (LMWH) is needed upon admission to hospital. Specific and sensitive rapid point of care (POC) tests allow important medical decisions to be made immediately in these emergency situations (-Fig. 1). POC INR tests can determine the intensity of VKA anticoagulation. ${ }^{8}$ The available methods for detecting DOACs may be limited by the presence of acute phase proteins and varying platelet counts in COVID-19 patients. $^{9}$ The DOAC Dipstick (Doasense $\mathrm{GmbH}$ ) is a rapid and accurate POC test that can be used on urine samples and is a viable option in emergency situations despite some limitations. ${ }^{10}$

Following discharge, COVID-19 patients will need to switch back from LMWH or heparin treatment to VKA or DOAC treatment if there is a clear indication for long-term anticoagulation. The effects of the antithrombin-dependent anticoagulants LMWH and heparin and the oral anticoagulants DOAC and VKA should be determined regularly, and these anticoagulants should be administered according to the reported dosing regimens using specific coagulation or chromogenic test system. ${ }^{11}$

(c) 2020. Thieme. All rights reserved. Thieme Medical Publishers, Inc., 333 Seventh Avenue, 18th Floor, New York, NY 10001, USA
DOI https://doi.org/ 10.1055/s-0040-1715091. ISSN 0094-6176. 


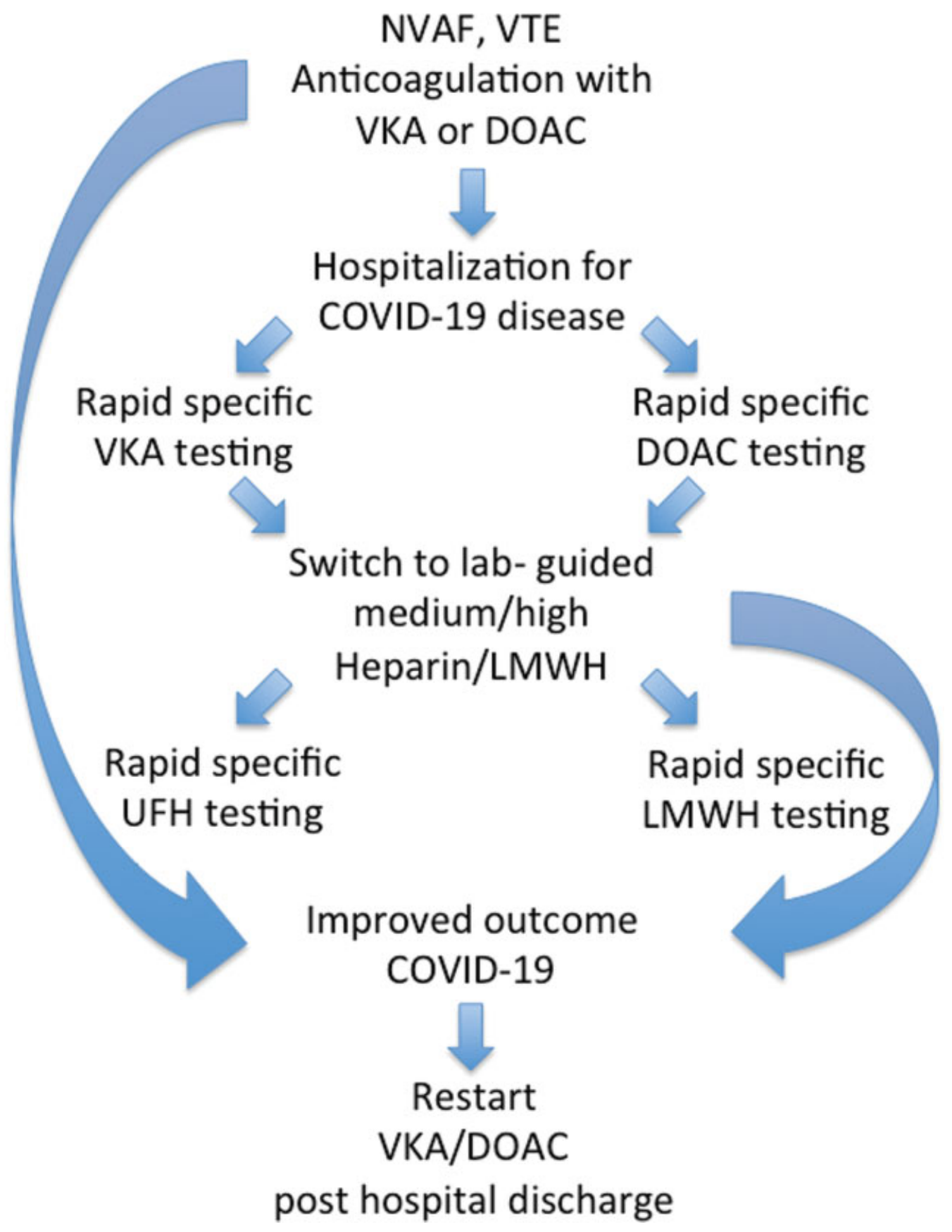

Fig. 1 Suggested effects of prehospital oral anticoagulation on outcome in COVID-19 patients. COVID-19, coronavirus disease 20019; DOAC, direct oral anticoagulant; LMWH, low molecular weight heparin; NVAF, nonvalvular atrial fibrillation; VKA, vitamin K antagonist; VTE, venous thromboembolism; UFH, unfractionated heparin.

In conclusion, we note that the number of NVAF and VTE patients with severe COVID-19 symptoms is very low; this may be explained by anticoagulant and nonanticoagulant effects of chronic treatment with DOAC or VKA, indicating a beneficial effect of prehospital oral anticoagulation and inhospital heparinization (-Fig. 1). However, specific analyses of available clinical studies are required to validate the interactions.

Conflict of Interest

None declared.

\section{References}

1 Wolf PA, Abbott RD, Kannel WB. Atrial fibrillation as an independent risk factor for stroke: the Framingham Study. Stroke 1991;22 (08):983-988

2 Lip GYH, Collet JP, de Caterina R, et al. Antithrombotic Therapy in Atrial Fibrillation Associated with Valvular Heart Disease: Executive Summary of a Joint Consensus Document from the European Heart Rhythm Association (EHRA) and European Society of Cardiology Working Group on Thrombosis, Endorsed by the ESC Working Group on Valvular Heart Disease, Cardiac Arrhythmia Society of Southern Africa (CASSA), Heart Rhythm Society (HRS), Asia Pacific Heart Rhythm Society (APHRS), South African Heart (SA Heart) Association and Sociedad Latinoamericana de 
Estimulación Cardíaca y Electrofisiología (SOLEACE). Thromb Haemost 2017;117(12):2215-2236

3 Rosendaal FR, VAN Hylckama Vlieg A, Doggen CJ. Venous thrombosis in the elderly. J Thromb Haemost 2007;5(Suppl 1):310-317

4 Zhou F, Yu T, Du R, et al. Clinical course and risk factors for mortality of adult inpatients with COVID-19 in Wuhan, China: a retrospective cohort study. Lancet 2020;395(10229):1054-1062

5 Liang W, Liang H, Ou L, et al; China Medical Treatment Expert Group for COVID-19. Development and validation of a clinical risk score to predict the occurrence of critical illness in hospitalized patients with COVID-19. JAMA Intern Med 2020 (e-pub ahead of print). Doi: 10.1001/jamainternmed.2020.2033

6 Alger HM, Williams JH IV, Walchok JG, Bolles M, Fonarow GC, Rutan C. Role of data registries in the time of COVID-19. Circ Cardiovasc Qual Outcomes 2020;13(05):e006766

7 Favaloro EJ, McVicker W, Lay M, et al. Harmonizing the International Normalized Ratio (INR): Standardization of methods and use of novel strategies to reduce interlaboratory variation and bias. Am J Clin Pathol 2016;145(02):191-202

8 Refaai MA, Shah V, Fernando R. Performance of the microINR Point-of-Care system: a multicenter clinical trial. Thromb Haemost 2020;120(04):687-691

9 Henry BM, de Oliveira MHS, Benoit S, et al. Hematologic, biochemical and immune biomarker abnormalities associated with severe illness and mortality in coronavirus disease 2019 (COVID19): a meta-analysis. Clin Chem Lab Med 2020;58(07): 1021-1028

10 Harenberg J, Beyer-Westendorf J, Crowther M, et al; Working Group Members. Accuracy of a rapid diagnostic test for the presence of direct oral factor Xa or thrombin inhibitors in urine-a multicenter trial. Thromb Haemost 2020;120(01):132-140

11 Lip GYH, Banerjee A, Boriani G, et al. Antithrombotic therapy for atrial fibrillation: CHEST guideline and expert panel report. Chest 2018;154(05):1121-1201 